\title{
Veno-arterial extracorporeal membrane oxygenation using Levitronix centrifugal pump as bridge to decision for refractory cardiogenic shock
}

\author{
Claudio F. Russo, MD, Aldo Cannata, MD, Marco Lanfranconi, MD, Giuseppe Bruschi, MD, \\ Filippo Milazzo, MD, Roberto Paino, MD, and Luigi Martinelli, MD
}

\begin{abstract}
Objectives: Cardiogenic shock still carries a very high mortality. We adopted veno-arterial extracorporeal membrane oxygenation using the Levitronix centrifugal pump (Levitronix LLC, Waltham, Massachusetts) as a firstline treatment of cardiogenic shock in a "bridge to decision" strategy. This article provides our experience of this clinical approach.
\end{abstract}

\begin{abstract}
Methods: Since 1988, 160 ventricular assist devices have been implanted at our hospital for heart failure. Since 2005, 15 consecutive patients have been treated with veno-arterial extracorporeal membrane oxygenation for refractory cardiogenic shock. Veno-arterial extracorporeal membrane oxygenation has been implanted either centrally or peripherally.
\end{abstract}

\begin{abstract}
Results: Mean age was $44.7 \pm 20.0$ years ( $2-78$ years). There were 5 women. Veno-arterial extracorporeal membrane oxygenation was implanted peripherally in 8 cases $(53.4 \%)$ and centrally in the remaining 7 (46.6\%). Mean veno-arterial extracorporeal membrane oxygenation duration was $11.5 \pm 8.1$ days (range, $1-30$ ). No patient experienced any neurologic event or vascular complication at the cannulation site. Twelve patients $(80 \%)$ were weaned from veno-arterial extracorporeal membrane oxygenation or bridged to either a long-term left ventricular assist device or heart transplantation. Three patients died during veno-arterial extracorporeal membrane oxygenation support secondary to multi-organ failure. Seven patients $(46.6 \%)$ were discharged from the hospital, with a $100 \%$ survival at follow-up. The survivors include 2 patients affected by fulminant myocarditis, who were bridged to recovery, and 5 patients who were bridged to heart transplantation. Survivors were younger than nonsurvivors (mean age, 28.5 vs 58.8 years, respectively).
\end{abstract}

Conclusions: In our experience, the use of veno-arterial extracorporeal membrane oxygenation as bridge to decision has been effective to promptly restore adequate systemic perfusion, allowing further time to evaluate myocardial recovery or candidacy for ventricular assist device or heart transplantation. Younger patients, with no or mild end-organ injury, had the best outcomes. Peripheral cannulation decreases the surgical trauma and makes emergency implantation possible, even in the intensive care unit. (J Thorac Cardiovasc Surg 2010;140:1416-21)

Refractory cardiogenic shock (RCS) still carries a very high mortality. ${ }^{1}$ In some cases of RCS mechanical circulatory support (MCS) represents the only therapeutic tool to rescue the patient's life if an intra-aortic balloon pump (IABP) is not able to provide sufficient cardiac support. However, uncertainty of patient clinical conditions and prognosis (ie, reversibility of myocardial damage, endorgan failure, and possible neurologic impairment), in addition to the time-pressure imposed by the severity of RCS, as well as logistical issues (ie, transportability of the patient

From A De Gasperis Cardiology \& Cardiac Surgery Department, Niguarda Ca' Granda Hospital, Milan, Italy

Disclosures: None.

Received for publication Dec 12, 2009; revisions received July 1, 2010; accepted for publication July 30, 2010; available ahead of print Oct 8, 2010.

Address for reprints: Claudio F. Russo, MD, Angelo De Gasperis Cardiology \& Cardiac Surgery Department, Niguarda Ca' Granda Hospital, Piazza Ospedale Maggiore, 3, Milan, 20162 Italy (E-mail: claudio.russo@ospedaleniguarda.it). $0022-5223 / \$ 36.00$

Copyright (c) 2010 by The American Association for Thoracic Surgery doi:10.1016/j.jtcvs.2010.07.083 and availability of an operating room), makes the proper selection of MCS more complex.

At Niguarda Hospital, experience with MCS started in 1988: until July 2009, 160 implants of monoventricular or biventricular ventricular assist device (VAD) have been performed. Since 2005, in severely ill patients with RCS requiring emergency MCS, we have been using veno-arterial extracorporeal membrane oxygenation (VA-ECMO) using the Levitronix CentriMag centrifugal pump (Levitronix LLC, Waltham, Mass) as a "bridge to decision." In this article, we review our experience in this clinical setting.

\section{MATERIALS AND METHODS}

We retrospectively reviewed our experience with VA-ECMO for RCS since 2005. The study was approved by the Hospital Review Board. Between February 2005 and July 2009, 15 consecutive patients were treated with VA-ECMO, using a Levitronix CentriMag Blood Pumping System (Levitronix LLC) as first-line emergency mechanical support for RCS. All patients were on maximal medical therapy, including at least two high-dose inotropes, mechanical ventilation, and IABP, if feasible (Table 1). Profound, rapidly progressing ventricular dysfunction with 


\section{Abbreviations and Acronyms \\ ACT = activated coagulation time \\ BSA = body surface area \\ $\mathrm{CS} \quad=$ Cardiogenic shock \\ HTx = heart transplant \\ IABP = intra-aortic balloon pump \\ $\mathrm{LV} \quad=$ left ventricle \\ LVAD $=$ left ventricular assist device \\ RCS = refractory cardiogenic shock \\ MCS = mechanical circulatory support \\ $\mathrm{VAD}=$ ventricular assist device \\ VA-ECMO $=$ veno-arterial extracorporeal membrane oxygenation}

persistent low blood pressure (systolic blood pressure $\leq 80 \mathrm{~mm} \mathrm{Hg}$ for adults; $\leq 60 \mathrm{~mm} \mathrm{Hg}$ for children) as measured by arterial lines and oliguria ( $\leq 0.5 \mathrm{~mL} / \mathrm{kg} / \mathrm{hr}$ ) for at least 4 hours despite maximal intropic support and IABP, which were promptly considered to be an indication for emergent VA-ECMO implantation. When the implantation was performed in the intensive care unit, all of the ECMO setup was obtained from the operating room. The Levitronix CentriMag Blood Pumping System is an extracorporeal short-term VAD composed of a single-use centrifugal blood pump, a motor, a console, and a flow probe. The CentriMag motor is based on bearingless technology that combines the drive, magnetic bearing, and rotor into a single unit. This system can generate flows up to $10 \mathrm{~L} /$ minute with a priming volume of $31 \mathrm{~mL}$, and in Europe the system is licensed for use for 30 days. Alongside the Levitronix CentriMag, we assembled an ECMO circuit that included a hollow fiber membrane oxygenator, the Quadrox D (Maquet, Hirrlingen, Germany), and a heater-cooler (Stockert, Munchen, Germany). If anatomically feasible, a femoral approach was our first choice for implantation. For femoral cannulation, we adopted percutaneous arterial (16-20 French) and venous (20-22 French) cannulas (Edwards Lifescience, Irvine, Calif). In 1 case, due to severe femoral artery disease, the axillary artery was cannulated. Patients were given a bolus of 100 $\mathrm{UI} / \mathrm{kg}$ of heparin, and after surgical dissection, the proximal common femoral vessels were cannulated. Only in 1 case we inserted the femoral cannulas percutaneously. As described elsewhere, ${ }^{3}$ to allow both to perfuse and drain of the distal limb, two 6-mm right-angled soft balloon-tipped coronary cannulas for selective anterograde cardioplegia infusion (Maquet, Hirrlingen, Germany) were inserted, respectively, into the common femoral artery and vein, distally to the main cannulation sites, and secured by pursestring sutures. Such small cannulas were connected to three-way stopcocks to allow both to perfuse and drain the distal limb. The clinical status of the limb-skin color and temperature (presence of edema) was evaluated every hour. To prevent thrombosis of distal venous drainage line, it was flushed with heparinized saline every 2 hours. In case of unfeasibility of femoral cannulation, because of small or severely atherosclerotic vessels, or in cases of postcardiotomy shock, we performed a central implant through standard pursestring sutures placed on the aortic arch and right atrium. In such case, we adopted a standard aortic cannula (20-22 French; Edwards Lifescience) and a 2-stage (36/46 French) venous cannula (NGC Medical Spa, Italy). The speed of the pump was then increased to provide a pump flow greater than $2.2 \mathrm{~L} / \mathrm{min} / \mathrm{m}^{2}$. Echocardiography was used to assess cardiac anatomy, ventricular function, and proper venous cannula placement. We used an $\mathrm{IABP}^{4}$ and low-dose epinephrine ${ }^{5}$ to reduce afterload and increase ventricular contractility to unload the left ventricle (LV). Heparin was infused to maintain an ACT of 160 to 180 seconds for the first 4 days to reduce the incidence of bleeding from the implantation site. After day 4, heparin dosing was increased to maintain an ACT of 180 to $200 \mathrm{sec}-$ onds. Close monitoring of hemostasis was performed by means of serial thromboelastography. End-organ function was closely monitored. Appropriate enteral or parenteral nutrition was used.

\section{Weaning From ECMO}

In cases in which recovery was expected (fulminant myocarditis or acute myocardial infarction), weaning was usually first attempted after 4 days of circulatory support, with normal end-organ function. The VAECMO flow was reduced to $500 \mathrm{~mL} /$ minute, and provided the $\mathrm{LV}$ ejection fraction (EF) of $\geq 40 \%$ and adequate hemodynamic parameters that were maintained with low-dose inotropes, from which the patient was weaned from support. However the weaning trial was discontinued in the case of unsatisfactory cardiac performance and a new attempt was made the following day.

\section{RESULTS}

Preoperative and mechanical support data for each patient are reported in Tables 1 and 2. Mean age was $44.7 \pm$ 20.0 years (2-78 years) and there were 5 females $(33.3 \%)$. The VA-ECMO was implanted peripherally in 8 cases $(53.4 \%)$ and centrally in the remaining 7 (46.6\%). In 5 cases the implant was performed in the intensive care unit. In 1 case $(6.6 \%)$, we resorted to direct left atrial venting to effectively decompress the left ventricle. Mean time on ECMO was $11.5 \pm 8.1$ days (range, $1-30$ ). Full circulatory support was achieved in all patients, with flows of up to $5.2 \mathrm{~L} /$ minute. Three patients were extubated while undergoing VA-ECMO through femoral cannulation. Overall preoperative data and outcomes are reported in Table 3. Bleeding requiring reoperation or more than 6 units of blood occurred in 4 patients $(26.6 \%)$ who had a gross coagulopathy. Two patients experienced pulmonary infections, as diagnosed by culture of bronchial secretions and radiologic evidence. No patient experienced any neurologic clinical event, defined as a neurologic deficit permanent or persistent for more than 24 hours, documented by means of computed tomography or at necropsy. We did not observe any mechanical device failure or mechanical hemolysis (plasma-free hemoglobin $>50 \mathrm{mg} / \mathrm{L}$ ). Twelve patients $(80 \%)$ were weaned from VA-ECMO or bridged to left ventricular assist device (LVAD) or heart transplant (HTx). Three patients died (20\%) during ECMO support.

Seven patients $(46.6 \%)$ were discharged from the hospital, with a $100 \%$ survival at follow-up (mean duration of follow-up, 26.1 months). The VA-ECMO successfully bridged to recovery 2 patients suffering from fulminant myocarditis, while the other 5 were bridged to HTx. Survivors were younger than nonsurvivors (mean age, 28.5 vs 58.8 years, respectively) without any differences in length of ECMO support (mean support, 11 vs 12 days, respectively). Prior to VA-ECMO implantation, both mean serum creatinine and total bilirubin were higher in nonsurvivors $(1.3 \pm 0.4 \mathrm{mg} / \mathrm{dL}$ and $1.5 \pm 1.0 \mathrm{mg} / \mathrm{dL}$, respectively), as compared to survivors $(1.0 \pm 0.5 \mathrm{mg} / \mathrm{dL}$ and $1.3 \pm 0.9$ $\mathrm{mg} / \mathrm{dL}$, respectively). The cause of in-hospital death was multiorgan failure in 6 of 8 cases $(75 \%)$, pulmonary artery 
TABLE 1. Patient characteristics before VA-ECMO implantation

\begin{tabular}{|c|c|c|c|c|c|c|c|c|}
\hline Patient & Gender & Age & BSA & Etiology & Serum creatinine & Total bilirubin & IABP & MV \\
\hline 1 & $\mathrm{~F}$ & 34 & 1.59 & Fulminant myocarditis & 0.7 & 0.6 & $\mathrm{Y}$ & $\mathrm{Y}$ \\
\hline 2 & M & 59 & 1.9 & Acute cardiac graft failure & 1.0 & 0.7 & $\mathrm{Y}$ & $\mathrm{Y}$ \\
\hline 3 & M & 65 & 1.97 & Acute myocardial infarction & 2.0 & 1.3 & $\mathrm{Y}$ & $\mathrm{Y}$ \\
\hline 4 & $\mathrm{~F}$ & 43 & 1.53 & Hypertrophic cardiomyopathy & 0.6 & 0.8 & $\mathrm{~N}$ & $\mathrm{Y}$ \\
\hline 5 & M & 78 & 1.9 & Postcardiotomy (MVR) & 1.2 & 1.4 & Y & $\mathrm{Y}$ \\
\hline 6 & $\mathrm{~F}$ & 63 & 1.67 & Postcardiotomy (VSR repair) & 1.3 & 0.9 & $\mathrm{Y}$ & $\mathrm{Y}$ \\
\hline 7 & M & 48 & 1.86 & Idiopathic dilated cardiomyopathy & 1.3 & 1.4 & $\mathrm{Y}$ & $\mathrm{Y}$ \\
\hline 8 & M & 33 & 1.93 & Ischemic cardiomyopathy & 0.9 & 2.4 & $\mathrm{Y}$ & $\mathrm{Y}$ \\
\hline 9 & M & 49 & 1.98 & Postcardiotomy (CABG) & 1.4 & 3.7 & $\mathrm{Y}$ & $\mathrm{Y}$ \\
\hline 10 & M & 42 & 1.57 & Idiopathic dilated cardiomyopathy & 0.6 & 2.5 & $\mathrm{Y}$ & $\mathrm{Y}$ \\
\hline 11 & M & 39 & 1.8 & LVAD thrombosis & 1.2 & 1.6 & $\mathrm{~N}$ & $\mathrm{Y}$ \\
\hline 12 & $\mathrm{~F}$ & 17 & 1.65 & Idiopathic dilated cardiomyopathy & 0.7 & 2.8 & $\mathrm{Y}$ & $\mathrm{Y}$ \\
\hline 13 & $\mathrm{~F}$ & 2 & 0.5 & Fulminant myocarditis & 0.8 & 0.8 & $\mathrm{~N}$ & $\mathrm{Y}$ \\
\hline 14 & M & 67 & 1.8 & Postinfarction VSR, s/p CABG & 1.9 & 0.7 & $\mathrm{Y}$ & $\mathrm{Y}$ \\
\hline 15 & M & 32 & 2.1 & Acute myocardial infarction & 2.1 & 0.4 & $\mathrm{Y}$ & $\mathrm{Y}$ \\
\hline
\end{tabular}

BSA is expressed as $\mathrm{m}^{2}$. Serum creatinine and total bilirubin are expressed as mg/dL. $B S A$, Body surface area; $C A B G$, coronary artery bypass grafting; $F$, female; $I A B P$, intraaortic balloon pump; $L V A D$, left ventricular assist device; $M$, male; $M V$, mechanical ventilation; $M V R$, mechanical ventilation; $N$, no; $V A-E C M O$, veno-arterial extracorporeal membrane oxygenation; $V S R, s / p C A B G$, ventricular septal rupture in previous CABG; $Y$, yes.

thrombosis in one case, and fatal thoracic bleeding complicating chest tube insertion in the remaining one. Just before ECMO support, both mean serum creatinine and total bilirubin were higher in patients who went on to develop multi-organ failure $(1.37 \mathrm{mg} / \mathrm{dL}$ and $1.72 \mathrm{mg} / \mathrm{dL}$, respectively) as compared to remaining patients $(1.05 \mathrm{mg} / \mathrm{dL}$ and $1.31 \mathrm{mg} / \mathrm{dL}$, respectively). Necropsy was performed in 6 cases. In every case, the pathologic findings confirmed the clinical diagnosis of cause of death. Regarding thromboembolic complications, multiple embolic renal infarctions were present in 2 patients, thrombosis of small branches of the pulmonary arteries in 2, massive thrombosis of the pulmonary artery trunk and multiple pulmonary infarctions in 1, and splenic infarction in 1. At necropsy, the brain was examined in only 2 patients, with evidence of mild edema without ischemic or hemorrhagic lesions.

\section{DISCUSSION}

Today RCS remains burdened by a very high mortality rate, despite the use of maximal pharmacologic therapy, IABP, mechanical ventilation, and continuous veno-venous hemofiltration. In this setting of severely ill patients, VADs represent a possible option, either as a bridge to recovery or HTx.

Unfortunately, mechanical support is not always live saving. Often the extreme severity and irreversibility of end-organ dysfunction can not be reversed, despite adequate circulatory support, making VAD implant useless and cost ineffective in these cases. In the most severe scenarios, the chances of recovery are often impossible to predict at presentation. Although severity of preoperative renal and liver injury is related to mortality on MCS, as reported by other authors, ${ }^{6}$ definite laboratory cut-offs are not yet available to discriminate consistently between reversible and irreversible end-organ injury. ${ }^{7}$ The etiology of cardiogenic shock is extremely variable, ranging from postcardiotomy shock to acute decompensation of chronic heart failure or acute onset of de novo cardiac failure. The patients in RCS can be very different from each other and their conditions are quite variable, and both are related to the previous health status as well as the duration of cardiogenic shock. Moreover, some patients can be comatose at presentation making any neurologic assessment very difficult. ${ }^{8}$ For these reasons the decision making in terms of timing of implant, type of support (monoventricular or biventricular) and device selection in such critically ill patients is quite difficult.

In the very acute scenario, VA-ECMO has several advantages in comparison to more complex circulatory mechanical support such as biventricular VAD. The implantation of a VAD requires an operating room. Even the transport from the emergency room of these patients on mechanical ventilation, inotropes, and IABP can be very risky and time consuming. Moreover, the significant surgical trauma associated with VAD implantation has a negative influence on postoperative mortality and morbidity in very sick patients. ${ }^{8,9}$

The ECMO allows for quick institution of full cardiopulmonary support independent to the extent of cardiac dysfunction (monoventicular or biventricular), stabilizes the hemodynamic status, and possibly promotes myocardial recovery. From a technical point of view femoral cannulation, when feasible, affords quick VA-ECMO implantation in the intensive care unit or emergency room. Furthermore, the surgical trauma of femoral cannulation is less when compared with VAD implantation: the chest is left untouched, reducing the risk of postoperative bleeding and infection 
TABLE 2. VA-ECMO support data and outcome

\begin{tabular}{|c|c|c|c|c|c|c|c|c|}
\hline Patient & $\begin{array}{c}\text { Implant } \\
\text { site } \\
\end{array}$ & $\begin{array}{c}\text { Pump } \\
\text { speed (rpm) } \\
\end{array}$ & $\begin{array}{c}\text { Pump } \\
\text { flow }(\mathrm{L} / \mathrm{min})\end{array}$ & $\begin{array}{c}\text { ECMO } \\
\text { duration (days) }\end{array}$ & $\begin{array}{c}\text { Weaned } \\
\text { from ECMO }\end{array}$ & $\begin{array}{l}\text { Bridge } \\
\text { to HTx }\end{array}$ & $\begin{array}{c}\text { Bridge to } \\
\text { LVAD } \\
\end{array}$ & $\begin{array}{r}\text { Hospital } \\
\text { discharge }\end{array}$ \\
\hline 1 & $\mathrm{P}$ & 4000 & 4.1 & 6 & $\mathrm{Y}$ & & & $\mathrm{Y}$ \\
\hline 2 & $\mathrm{C}$ & 3700 & 3.6 & 7 & $\mathrm{~N}$ & & & $\mathrm{~N}$ \\
\hline 3 & $\mathrm{C}$ & 3300 & 4.3 & 18 & & & $\mathrm{Y}$ & $\mathrm{N}$ \\
\hline 4 & $\mathrm{C}$ & 3600 & 4.6 & 6 & & $\mathrm{Y}$ & & $\mathrm{Y}$ \\
\hline 5 & $\mathrm{C}$ & 4800 & 4.0 & 4 & $\mathrm{Y}$ & & & $\mathrm{N}$ \\
\hline 6 & $\mathrm{C}$ & 4200 & 4.8 & 4 & & $\mathrm{Y}$ & & $\mathrm{N}$ \\
\hline 7 & $\mathrm{P}$ & 4000 & 4.2 & 16 & & & $\mathrm{Y}$ & $\mathrm{N}$ \\
\hline 8 & $\mathrm{P}$ & 3700 & 4.4 & 24 & & $\mathrm{Y}$ & & $\mathrm{Y}$ \\
\hline 9 & $\mathrm{P}$ & 3900 & 3.9 & 13 & $\mathrm{~N}$ & & & $\mathrm{~N}$ \\
\hline 10 & $\mathrm{P}$ & 3800 & 4.4 & 4 & $\mathrm{~N}$ & & & $\mathrm{~N}$ \\
\hline 11 & $\mathrm{P}$ & 4400 & 5.2 & 13 & & $\mathrm{Y}$ & & $\mathrm{Y}$ \\
\hline 12 & $\mathrm{C}$ & 3700 & 4.0 & 1 & & $\mathrm{Y}$ & & $\mathrm{Y}$ \\
\hline 13 & $\mathrm{C}$ & 2600 & 2.5 & 30 & $\mathrm{Y}$ & & & $\mathrm{Y}$ \\
\hline 14 & $\mathrm{P}$ & 3900 & 4.0 & 12 & $\mathrm{Y}$ & & & $\mathrm{N}$ \\
\hline 15 & $\mathrm{P}$ & 4200 & 4.5 & 15 & & $\mathrm{Y}$ & & $\mathrm{Y}$ \\
\hline
\end{tabular}

$C$, Central; $E C M O$, extracorporeal membrane oxygenation; $H T x$, heart transplantation; $L V A D$, left ventricular assist device; $N$, no; $P$, peripheral; $V A-E C M O$, venoarterial extracorporeal membrane oxygenation; $Y$, yes; $N$, no.

following an eventual VAD implantation or heart transplantation. Finally, when feasible, weaning from mechanical ventilation is easier and safer in case of peripheral cannulation as compared to central cannulation, as we observed in 3 patients.

Prompt VA-ECMO implant guarantees an extended "window of observation" in stable circulatory conditions. According to others, ${ }^{8}$ the stabilization of the patient should be the primary goal, restoring systemic perfusion with minimal risk of complications while preventing malperfusion damage. During support, the end-organ function, the neurologic status, the chance of myocardial recovery or the indication to move to further therapies (ie, VAD or HTx) can be adequately assessed. ${ }^{2,8}$ The ECMO in RCS appears crucial for a more rational use of such limited resources, such as HTx and VAD, by avoiding their adoption in very highrisk patients who are not going to survive, even on ECMO, because of severe and irreversible preimplant shock sequelae. $^{2,6}$

During the recent years, with improving equipment and increased experience, ECMO technology has become more reliable with improved results. ${ }^{10-12}$ Particularly, magnetically suspended pumps minimize blood trauma $^{13,14}$ and have a very low incidence of mechanical failure. The Levitronix centrifugal pump is easy to prime, implant, and manage. It is cheaper than more complex VADs and reliable for support longer than 6 weeks, without device-related complications. ${ }^{15,16}$ Our experience with Levitronix Centrimag confirms its reliability and the reported satisfactory outcomes. ${ }^{15-18}$

When it comes to surgical aspects, the peripheral approach, instead of a central one, is well suited and should be considered first in most patients, with the exception of those with small femoral vessels or in postcardiotomy shock.

According to other studies, ${ }^{2,6,19}$ and in our experience, refractory cardiogenic shock carries still a high hospital mortality, despite of prompt institution of MCS support. The mortality reflects the very sick conditions of these patients. In our series, among survivors there were 2 cases of fulminant myocarditis and 5 cases successfully bridged to HTx. However, prognosis in VA-ECMO for postcardiotomy shock was dismal. Such observation confirms the results reported from other groups. ${ }^{5,19}$ In this study, age and aetiology were both major determinants of outcome. Particularly patients with potentially recoverable conditions, such as fulminant myocarditis and patients who did not have any underlying condition, which would contraindicate HTx had better survival outcomes. Survivors were younger than nonsurvivors (mean age, 28.5 vs 58.8 years, respectively). Preimplant markers of renal and liver injury were slightly higher in nonsurvivors. Twelve of 15 patients $(80 \%)$ were weaned from VAECMO or bridged to LVAD/HTx. The mean duration of VA-ECMO was 11 days, and we did not observe any neurologic complication or device failure during this time. The most common cause of death was multi-organ failure, which occurred despite restoration of adequate systemic blood flow. In these cases, the extreme severity of endorgan dysfunction could not be reversed, even by adequate circulatory and respiratory support. As a result, due to the unpredictability of outcomes, in this group of patients we assumed that a more complex, invasive, and expensive MCS system should have not be considered the "first" support option. The VA-ECMO was implanted as a bridge to decision, and if necessary it was switched to the implantable 
TABLE 3. Preoperative data and outcome summary

\begin{tabular}{lcc}
\hline Mean age & $44.7 \pm 20.0$ years & $(2-78$ years) \\
Female & $5 / 15$ & $(33.3 \%)$ \\
Previous cardiac surgery & $2 / 15$ & $(13.3 \%)$ \\
Postcardiotomy LCOS & $3 / 15$ & $(20.1 \%)$ \\
Pre-ECMO inotropes* & $15 / 15$ & $(100 \%)$ \\
Pre-ECMO MV & $15 / 15$ & $(100 \%)$ \\
Pre-ECMO IABP & $12 / 15$ & $(80.0 \%)$ \\
Peripheral cannulation & $8 / 15$ & $(53.4 \%)$ \\
Central cannulation & $7 / 15$ & $(46.6 \%)$ \\
Direct LV venting & $1 / 15$ & $(6.6 \%)$ \\
Mean ECMO duration & $11.5 \pm 8.1$ days & $(1-30$ days) \\
Patients weaned from ECMO & $4 / 15$ & $(26.6 \%)$ \\
Patients bridged to HTx & $6 / 15$ & $(40.0 \%)$ \\
Patients bridged to LVAD & $2 / 15$ & $(13.3 \%)$ \\
Patients weaned or bridged to other & $12 / 15$ & $(80.0 \%)$ \\
Mortality on ECMO & $3 / 15$ & $(20.1 \%)$ \\
Patients discharged form the hospital & $7 / 15$ & $(46.6 \%)$ \\
\hline$E C M O$, Extracorporeal membrane oxygenation; $H T x$, heart & transplantation; \\
$I A B P$, intra-aortic balloon pump; $L C O S$, low cardiac output syndrome; $L V A D$, left \\
ventricular assist device; $L V$, left ventricle; $M V$, mechanical ventilation. *Intravenous \\
epinephrine, infusion rate more than 0.09 mcg/kg/min.
\end{tabular}

VAD and/or HTx in patients without cardiac recovery provided full hemodynamic, neurologic, and end-organ function recovery had taken place.

Technical solutions for potential drawbacks of peripherally implanted VA-ECMO deserve discussion. In small body surface area (BSA) patients, particularly young females, the diameter of femoral vessels can be too small for cannulation. In our experience, in only 2 cases (ie, 2 females with BSA, ranging between 1.5 and $1.6 \mathrm{~m}^{2}$ ) we proceeded to a central implant, following an initial attempt at peripheral cannulation, because of the small diameter of the femoral vessels. In 1 patient we cannulated the right axillary artery for arterial return because of severe atherosclerosis of the femoral vessels. An increased risk of early and late vascular complications following peripheral cannulation for VA-ECMO are reported. ${ }^{20,21}$ Huang and colleagues $^{22}$ suggested that a mean pressure less than $50 \mathrm{~mm} \mathrm{Hg}$ into the superficial femoral artery is an indication to the placement of a catheter for distal perfusion. However, little attention has been devoted to the risk of severe venous stasis following femoral vessels cannulation. Restoring the limb perfusion by means of an additional arterial cannula may even precipitate venous stasis, particularly if the venous cannula completely obstructs the distal common femoral vein. To avoid such a complication, we recommend our technique of distal limb perfusion and drainage during VA-ECMO. ${ }^{3}$ With this technique we did not observe any limb complications during peripheral VA-ECMO and no vascular reconstruction of the femoral vessels after decannulation was required.

Left ventricular decompression during VA-ECMO is of paramount importance to promote myocardial recovery and to prevent pulmonary edema. Retrograde arterial perfusion, aortic valve regurgitation, partial drainage of the right heart, and bronchial arterial flow are contributing factors in causing left ventriclular distension, particularly if the ventricle itself is unable to eject. Moreover, distension and blood stasis inside the left ventricle could lead to formation of thrombus that may embolize. For these reasons, some degree of left ventricular ejection should be maintained during support. Although not uniformly agreed, ${ }^{2}$ we recommend $\mathrm{IABP}^{4}$ and low-dose epinephrine ${ }^{5}$ to achieve left ventricle decompression, by promoting afterload reduction and increased contractility. Beneficial effects of IABP during nonpulsatile circulatory support have been reported., ${ }^{4,23}$ Moreover, diastolic pressure augmentation improves coronary perfusion, contributing to myocardial recovery. Serial echocardiographic monitoring of the left ventricle is mandatory to evaluate $\mathrm{LV}$ unloading during support.

Finally, we observed at autopsy several cases of pulmonary and systemic thromboembolism, similar to the experiences reported by Rastan and colleagues. ${ }^{24}$ In some instances, thromboembolism has been shown only at the autopsy. Clinical underestimation of the thromboembolic burden of MCS is very likely. In our series, despite the absence of neurologic clinical events, the real rate of cerebral embolism is probably underestimated because of the small number of brains examined at necropsy. We found macroscopic clots inside the circuit in 1 case; these were in the oxygenator. The pathogenesis of the thromboembolism is multifactorial (ie, blood activation following contact with foreign surfaces, blood stasis in the cardiac chambers and the systemic veins, disseminated intravascular coagulation, and pulmonary vascular disease), potentially contributing mechanisms. These findings underscore the compelling need for further studies on the prothrombotic state and appropriate coagulation management during mechanical circulatory support.

\section{CONCLUSIONS}

In our experience, the use of VA-ECMO as bridge to decision has been effective in promptly restoring adequate systemic perfusion, allowing further time to evaluate myocardial recovery or candidacy for ventricular assist device or heart transplantation. Younger patients with no or mild end-organ injury showed the best outcomes. Peripheral cannulation decreases the surgical trauma and makes emergency implantation possible, even in the intensive care unit. Appropriate timing of implant and proper management on support to avoid complications are warranted to improve results.

\section{References}

1. Carnendran L, Abboud R, Sleeper LA, Gurunathan R, Webb JG, Menon V, et al. Trends in cardiogenic shock: report from the SHOCK Study. Should we emergently revascularize occluded coronaries for cardiogenic shock? Eur Heart J. 2001;22:472-8. 
2. Hoefer D, Ruttmann E, Poelzl G, Kilo J, Hoermann C, Margreiter R, et al. Ann Thorac Surg. 2006;82:28-33.

3. Russo CF, Cannata A, Vitali E, Lanfranconi M. Prevention of limb ischemia and edema during peripheral venoarterial extracorporeal membrane oxygenation in adults. J Card Surg. 2009;24:185-7.

4. Doll N, Kiaii B, Borger M, Bucerius J, Krämer K, Schmitt DV, et al. Five-year results of 219 consecutive patients treated with extracorporeal membrane oxygenation for refractory postoperative cardiogenic shock. Ann Thorac Surg. 2004;77:151-7.

5. Chen YS, Yu HY, Huang SC, Chiu KM, Lin TY, Lai LP, et al. Experience and result of extracorporeal membrane oxygenation in treating fulminant myocarditis with shock: what mechanical support should be considered first? J Heart Lung Transplant. 2005;24:81-7.

6. Pagani FD, Lynch W, Swaniker F, Dyke DB, Bartlett R, Koelling T, et al. Extracorporeal life support to left ventricular assist device bridge to heart transplant: a strategy to optimize survival and resource utilization. Circulation. 1999;100: II206-10.

7. Wadia Y, Etheridge W, Smart F, Wood RP, Frazier OH. Pathophysiology of hepatic dysfunction and intrahepatic cholestasis in heart failure and after left ventricular assist device support. J Heart Lung Transplant. 2005;24: 361-70.

8. John R, Liao K, Lietz K, Kamdar F, Colvin-Adams M, Boyle A, et al. Experience with the Levitronix CentriMag circulatory support system as a bridge to decision in patients with refractory acute cardiogenic shock and multisystem organ failure. J Thorac Cardiovasc Surg. 2007;134:351-8.

9. Alba AC, Rao V, Ivanov J, Ross HJ, Delgado DH. Usefulness of the INTERMACS scale to predict outcomes after mechanical assist device implantation. J Heart Lung Transplant. 2009;28:827-33.

10. DeRose JJ Jr, Umana JP, Argenziano M, Catanese KA, Levin HR, Sun BC, et al. Improved results for postcardiotomy cardiogenic shock with the use of implantable left ventricular assist devices. Ann Thorac Surg. 1997;64:1757-63.

11. Combes A, Leprince P, Luyt CE, Bonnet N, Trouillet JL, Léger P, et al. Outcomes and long-term quality-of-life of patients supported by extracorporeal membrane oxygenation for refractory cardiogenic shock. Crit Care Med. 2008;36:1404-11.

12. Hoefer D, Ruttmann E, Poelzl G, Kilo J, Hoermann C, Margreiter R, et al. Outcome evaluation of the bridge-to-bridge concept in patients with cardiogenic shock. Ann Thorac Surg. 2006;82:28-33.
13. Nakazawa T, Makinouchi K, Ohara Y, Ohtsubo S, Kawahito K, Tasai K, et al. The effect of the impeller- driver magnetic coupling distance on hemolysis in a compact centrifugal pump. Artif Organs. 1996;20:252-7.

14. Göbel C, Eilers R, Reul H, Schwindke P, Jörger M, Rau G. A new blood pump for cardiopulmonary bypass: the Hiflow centrifugal pump. ArtifOrgans. 1997;21:841-5.

15. De Robertis F, Birks EJ, Rogers P, Dreyfus G, Pepper JR, Khagani A. Clinical performance with the Levitronix CentriMag short-term ventricular assist device. J Heart Lung Transplant. 2006;25:181-6.

16. De Robertis F, Rogers P, Amrani M, Petrou M, Pepper JR, Bahrami T, et al Bridge to decision using the Levitronix CentriMag short-term ventricular assist device. J Heart Lung Transplant. 2008;27:474-8.

17. Santise G, Petrou M, Pepper JR, Dreyfus G, Khaghani A, Birks EJ. Levitronix as a short-term salvage treatment for primary graft failure after heart transplantation. J Heart Lung Transplant. 2006;25:495-8.

18. John R, Liao K, Lietz K, Kamdar F, Colvin-Adams M, Boyle A, et al. Experience with the Levitronix CentriMag circulatory support system as a bridge to decision in patients with refractory acute cardiogenic shock and multisystem organ failure. J Thorac Cardiovasc Surg. 2007;134:351-8.

19. Bakhtiary F, Keller H, Dogan S, Dzemali O, Oezaslan F, Meininger D, et al. Venoarterial extracorporeal membrane oxygenation for treatment of cardiogenic shock: clinical experiences in 45 adult patients. J Thorac Cardiovasc Surg. 2008; $135: 382-8$

20. Zimpfer D, Heinisch B, Czerny M, Hoelzenbein T, Taghavi S, Wolner E, et al Late vascular complications after extracorporeal membrane oxygenation support. Ann Thorac Surg. 2006;81:892-5.

21. D'Alessandro C, Aubert S, Golmard JL, Praschker BL, Luyt CE, Pavie A, et al Extra-corporeal membrane oxygenation temporary support for early graft failure after cardiac transplantation. Eur J Cardiothorac Surg. 2010;37:343-9.

22. Huang SC, Yu HY, Ko WJ, Chen YS. Pressure criterion for placement of distal perfusion catheter to prevent limb ischemia during adult extracorporeal life support. J Thorac Cardiovasc Surg. 2004;128:776-7.

23. Madershahian N, Liakopoulos OJ, Wippermann J, Salehi-Gilani S, Wittwer T, Choi YH, et al. The impact of intraaortic balloon counterpulsation on bypass graft flow in patients with peripheral ECMO. J Card Surg. 2009;24:265-8.

24. Rastan AJ, Lachmann N, Walther T, Doll N, Gradistanac T, Gommert JF, et al Autopsy findings in patients on postcardiotomy extracorporeal membrane oxygenation (ECMO). Int J Artif Organs. 2006;29:1121-31. 